\title{
Beneficial effects of baicalein on a model of allergic rhinitis
}

\author{
TAO LIU $\mathrm{a}^{\mathrm{a}, 1,2}$ \\ JING XUa,2 \\ YUNGANG WU ${ }^{\mathrm{a}, 2}$ \\ XIAOXIA $\mathrm{LI}^{2}$ \\ DETAO DING ${ }^{2}$ \\ DENGDIAN MA ${ }^{2}$ \\ MENGWEI YAO ${ }^{2}$ \\ WENZHONG WEI ${ }^{2}$ \\ WEI ZHANG ${ }^{4}$ \\ SHAOHUA WANG 2 \\ JING YAO $3, *$ \\ XIAOYU LI $I^{1,2, *}$ \\ ${ }^{1}$ School of Clinical Medicine, Jining Medical \\ University, Jining, Shandong, China \\ ${ }^{2}$ Affiliated Hospital of Jining Medical \\ University, Jining, Shandong, China \\ ${ }^{3}$ School of Basic Medicine, Jining Medical \\ University, Jining, Shandong, China

\section{${ }^{4}$ People's Hospital of Hengshui, Hengshui} \\ Hebei, China
}

Accepted February 28, 2019

Published online May 16, 2019

\begin{abstract}
Allergic rhinitis (AR) is a common disease that causes severe inflammation and even disabilities. Previous studies have reported baicalein to have an anti-inflammatory effect. However, the pharmacological action of baicalein on anaphylaxis has not been clarified yet. This study assessed the in vivo protective effect of baicalein post-treatment in an ameliorating ovalbumin (OVA)-sensitized AR rat model. Baicalein attenuated histological alterations, aberrant tissue repair and inflammation after OVA-induced AR. Baicalein reduced the frequency of nasal/ear rubs and sneezes in rats, and inhibited generation of several inflammatory cytokines (TNF- $\alpha$, IL- $1 \beta$, and IL-6) in both blood and nasal lavage of rats. Infiltrations of eosinophils, lymphocyte, and neutrophils were decreased in baicalein-administered rats. Furthermore, baicalein inhibited the expression of STAT3 phosphorylation in the nasal mucosa. In summary, baicalein attenuated OVA-induced AR and inflammation, which suggests it as a promising therapeutic agent for the alleviation of AR-associated inflammation and pathology.
\end{abstract}

Keywords: baicalein, ovalbumin, allergic rhinitis, inflammatory factors, p-STAT3

Allergic rhinitis (AR) is a common allergic disease and an increasing health problem in different age groups $(1,2)$. Although AR is not life-threatening, it frequently co-occurs with asthma and atopic eczema, which have negative effects on the patients' quality of life, as well as their academic and job performances (3). AR causes severe pain and has several symptoms, such as nasal congestion, sneezing, nasal itching, respiratory obstruction, rhinorrhea and nasal overflow. Moreover, prolonged AR can lead to abnormalities in the nasal cavity, and severe ear symptoms (4). AR is a chronic disease of the upper respiratory tract caused by a complex allergen-induced inflammatory process, which causes inflammation of the nasal mucosa. Similar symptoms are caused by the release of histamine, along with several cytokines, various proinflammatory mediators and other active substances, which can induce vascular dilation, and cause secretion of tears (5). The synthesis and release of

\footnotetext{
*Correspondence, e-mail: 1xyent@163.com; yjing_87@163.com

a These three authors contributed equally to the paper.
} 
immunoglobulin E (IgE) play a central role in the allergic inflammatory process of $\mathrm{AR}$, and in the increase of eosinophils, basophils and mast cell numbers (6). Despite an abundance of research on the pathogenesis and symptoms of AR, current treatments cannot reduce the occurrence rate of AR. Therefore, innovative and effective strategies are still urgently needed for patients with AR. Animal AR models that can be induced by experimental administration of ovalbumin (OVA) have been extensively applied to test new drugs (7-9).

Flavonoids, the key bioactive components in Scutellariae radix, are composed of a group of natural and low-molecular-weight vegetal products, including oroxylin A, wogonin, baicalein and baicalin (10). These products are renowned for their diverse biological activities, such as anti-inflammatory activities. Baicalein has been reported to alleviate allergic asthma by adjusting the cytokine imbalance of Th1/Th2 and histamine release in mast cells (11). Increasing evidence shows that baicalein has an anti-inflammatory effect in mice, alleviating liver inflammation (12), vascular inflammation (13) and colitis induced by dextran sulfate sodium (DSS) and 2,4,6-trinitrobenzene sulfonic acid (TNBS) (14, 15), lipopolysaccharide-evoked acute lung inflammation (16), pancreatitis (17) and diet-induced non-alcoholic steatohepatitis in mice (18). In this study, a rat model of AR was used to investigate the regulatory activity of baicalein on the clinical symptoms of AR, mucosal histological changes, and inflammation in vivo.

\section{EXPERIMENTAL}

\section{Materials}

OVA (grade V) was obtained from Sigma (USA), aluminum hydroxide gel was obtained from Thermo (USA) and anti-rat IL-6/IL-1 $\beta /$ TNF- $\alpha$ enzyme-linked immunosorbent assay (ELISA) kits were obtained from Feiya Biotech (China). Baicalein was provided by Dr Qinglong Guo of the College of Pharmacy at China Pharmaceutical University. Antibodies against phospho-STAT3 (Tyr705) were obtained from Cell Signaling Technology (USA), which were used for immunohistochemical staining at 1:100 dilution. BCA Protein Assay kit was purchased from Thermo Fisher Scientific (USA). The kit for immunohistochemical staining was obtained from Keygen biotechnology Co., Ltd. (China). Diaminobenzidine (DAB) was obtained from Solarbio Science \& Technology Co., Ltd. (China). Hematoxylin and eosin were purchased from Solarbio Science \& Technology Co., Ltd. (China).

\section{Animals}

The experimental process was approved by the Committee for Ethical Use and Care of Animals of the Jining Medical University. Forty male Sprague-Dawley rats, weighing between $200 \mathrm{~g}$ and $220 \mathrm{~g}$, were obtained from Jinan Pengyue Experimental Animal Breeding Co. Ltd. (China). The rats were fed in an air-conditioned room at ambient temperature of $24 \pm 1{ }^{\circ} \mathrm{C}$ and relative humidity of $55 \pm 5 \%$, with a 12-hour light-dark cycle.

\section{OVA-induced AR model}

Rats were randomly assigned to the following four groups: (1) the saline group (negative control group, $n=10$ ) was composed of rats that were intranasally challenged with saline alone; (2) the OVA group (model group, $n=10$ ); (3) the OVA+baicalein group (drug 
group, $n=10$ ) was composed of rats that were sensitized, intranasally challenged with OVA and administered baicalein before daily intranasal challenge; (4) the OVA+clarityne group (positive control group, $n=10$ ), composed of rats that were sensitized, intranasally challenged with OVA and administered clarityne before daily intranasal challenge.

After acclimatization to laboratory conditions for one week, 30 rats were sensitized intraperitoneally with OVA $(0.3 \mathrm{mg})$ as antigen, in addition to aluminum hydroxide $(30 \mathrm{mg})$ in saline ( $1 \mathrm{~mL}$ intraperitoneally) every two days for 16 days. At stage two, all 30 sensitized rats were used to develop an AR model by repeating daily intranasal injections with $20 \mathrm{mg} \mathrm{mL}^{-1}$ OVA of $10 \mu \mathrm{L}$ via a micropipette for 21 days. The 10 rats in the OVA+baicalein group received an intragastric injection of $40 \mathrm{mg} \mathrm{kg}^{-1}$ baicalein dissolved in $0.9 \%$ physiological saline $30 \mathrm{~min}$ before OVA administration on the 17th day. In the OVA group, 10 rats were repeatedly sensitized and exposed to saline at the same dosage and at times similar to rats in the other two groups. In addition, the OVA+clarityne group rats were intragastrically injected with $50 \mathrm{mg} \mathrm{kg}^{-1}$ clarityne dissolved in $0.9 \%$ physiological saline 30 min before OVA administration. The rats were weighed daily and were monitored for nasal symptoms.

\section{Evaluation of symptoms}

The real-time and late-phase reactions of all nasally challenged rats were inspected on days $1,15,17,19,21,23,25,27,29,31,33,35$ and 37 after OVA administration. The frequency of nasal rubs, sneezing, and nasal secretion that occurred within $30 \mathrm{~min}$ after OVA i.n. provocation after challenge was observed, evaluated and recorded by an observer. Nasal symptoms were scored as a number (0-3) and graded scores are shown in Table I. An aggregate score exceeding five indicated a successful AR model (11).

\section{Collection of serum, nasal lavage fluid and major organs}

Rats of each group were administered $10 \%$ chloral hydrate $\left(300 \mathrm{mg} \mathrm{kg}^{-1}\right)$ after $24-\mathrm{h}$ stimulation. Rats were fixed on an operating table at an angle of about $45^{\circ}$, the abdominal skin was cut to expose the abdominal aorta, and blood was collected into prepared plastic tubes.

The nasal lavage fluid was collected by inserting an epidural catheter about $2 \mathrm{~cm}$ into one side of the nasal cavity. Then, $1.5 \mathrm{~mL}$ saline was slowly injected into the nasal cavity and the nasal outflow of liquid was received. The same process was performed on the other nasal cavity. In addition, the heart, liver, spleen, lung, and kidney tissues were extracted from the experimental animals. Finally, the main organs were weighed and the relevant coefficients were calculated to detect visceral injuries caused by baicalein.

\section{Enzyme-linked immunosorbent assay (ELISA)}

Cytokines IL-6, IL-1 $\beta$, and TNF- $\alpha$ in serum and nasal lavage fluid were detected by ELISA following the manufacturer's instructions. All tests were conducted in duplicate.

\section{Determination of protein concentration in nasal lavage fluid}

The remaining nasal lavage fluid was centrifuged at $1000 \times \mathrm{g}$ for $5 \mathrm{~min}$ at $4{ }^{\circ} \mathrm{C}$, and the cell-free supernatant was kept at $-80^{\circ} \mathrm{C}$ for the subsequent analysis of protein concentration via the BCA Protein Assay kit following the instructions provided by the manufacturer. 


\section{Histopathological evaluation}

Rats were killed $24 \mathrm{~h}$ after final intranasal challenge at day 38 . Their heads were fixed in $10 \%$ neutral-buffered formalin for $24 \mathrm{~h}$. Then, their nasal mucosa and nasal septum tissues were removed and embedded in paraffin, $4 \mu \mathrm{m}$ thin-sections were cut, and hematoxylin-eosin (H\&E) or immunohistochemically stained. Immunohistochemical staining against p-STAT3 was performed using standard techniques as previously described (19).

\section{Statistical analysis}

All results in the paper are expressed as means of \pm SD from parallel triple experiments, unless otherwise specified. Statistically significant differences (analysis of variance (ANOVA) and post hoc tests) were analyzed with GraphPad Prism software (GraphPad Software Inc., USA). Details of each statistical analysis are provided in figure legends. Differences with $p$ values $<0.05$ were considered statistically significant.
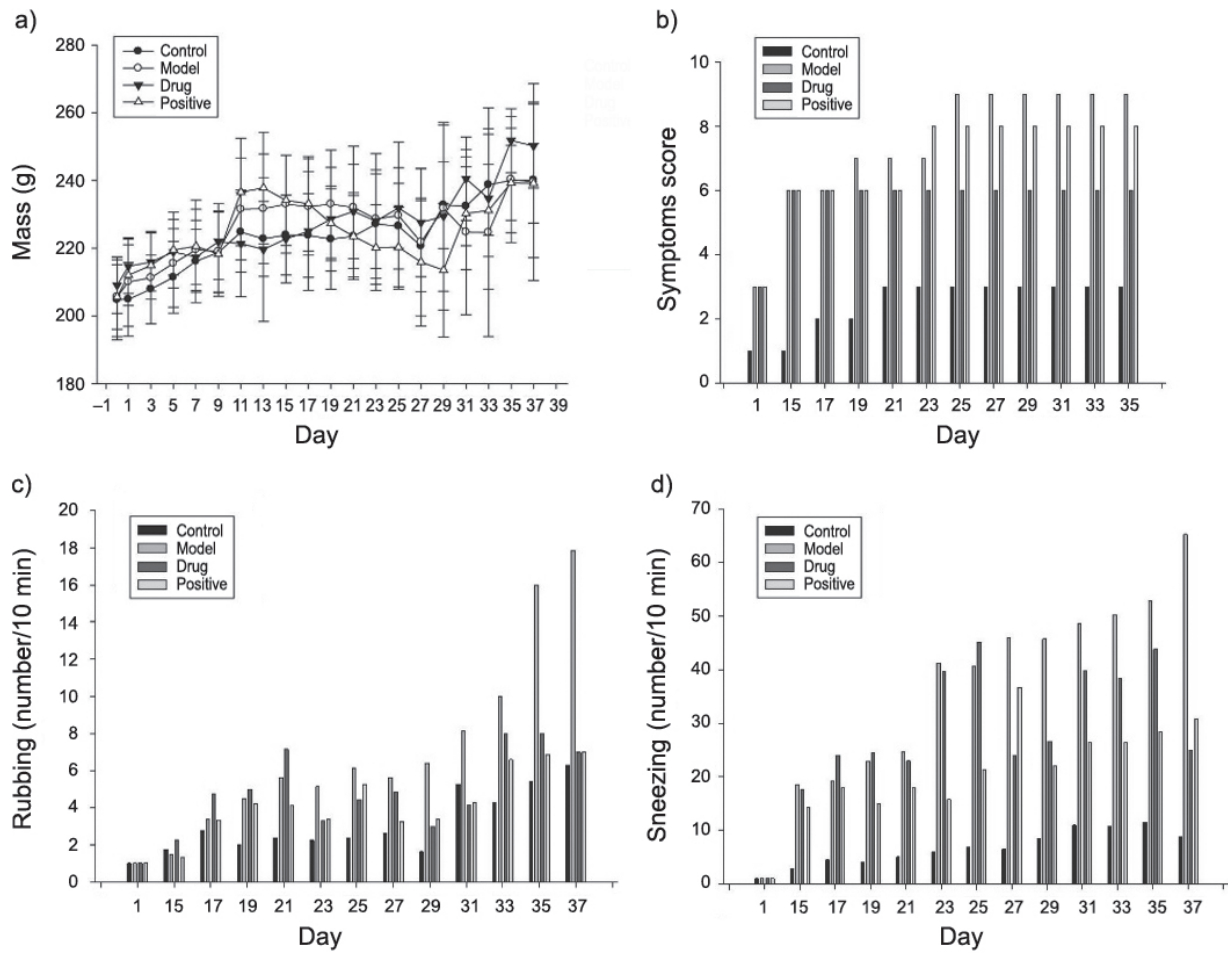

Fig. 1. Treatment with baicalein and clarityne relieved the symptoms in OVA-induced rats. a) Body weights of OVA group, OVA+baicalein group, and OVA+clarityne group were measured, b) scores of nasal symptoms within $30 \mathrm{~min}$ after the last administration in each group, $\mathrm{c}$ ) and d) show the numbers of nasal scratching and sneezing in OVA-sensitized rats $(n=10)$ and OVA+baicalein treated rats $(n=10)$. 


\section{RESULTS AND DISCUSSION}

\section{Therapeutic effect of baicalein on AR-induced rats}

Body weights of rats did not change significantly in the OVA group, the OVA+baicalein group and the OVA+clarityne group (Fig. 1a). After stimulation with OVA for $30 \mathrm{~min}$, the frequency of sneezing, nasal scratching and the degree of nasal outflow of rats were recorded in each group and then superimposed according to the applied score rule. Relevant results are shown in Fig. $1 \mathrm{~b}$. Since the total score of all rats exceeded 5, the AR model was treated as successful. It is noteworthy that the frequency of sneezing and nasal scratching after OVA challenge was higher than in the normal group, manifested as an outflow to the face. Moreover, the baicalein-treated groups showed lower frequency of sneezing and nasal scratching (Figs. 1c,d). The results showed the differences in the frequency of sneezing between the model group and the drug group for the periods of 1-25 days (no drug inhibition), 27-29 days (drug inhibition) and the 31st day (poor drug inhibition). The exact mechanism remains unclear. The disease could have recurrent symptoms, which counteracted the inhibitory effect of the drug on the disease progress; however, the results still showed baicalein-mediated inhibitory effect on the frequency of sneezing in general. These results showed that baicalein could inhibit the development of allergic rhinitis induced by OVA.

Masses of the main organs (including heart, liver, spleen, lung, and kidney) are shown in Fig. 2. The spleen in the model group was statistically significantly heavier than that in the control group $(p<0.05)$, while lighter in the baicalein-treated group compared to the model group $(p<0.05)$. The spleen is involved in the immune response to AR, which indi-
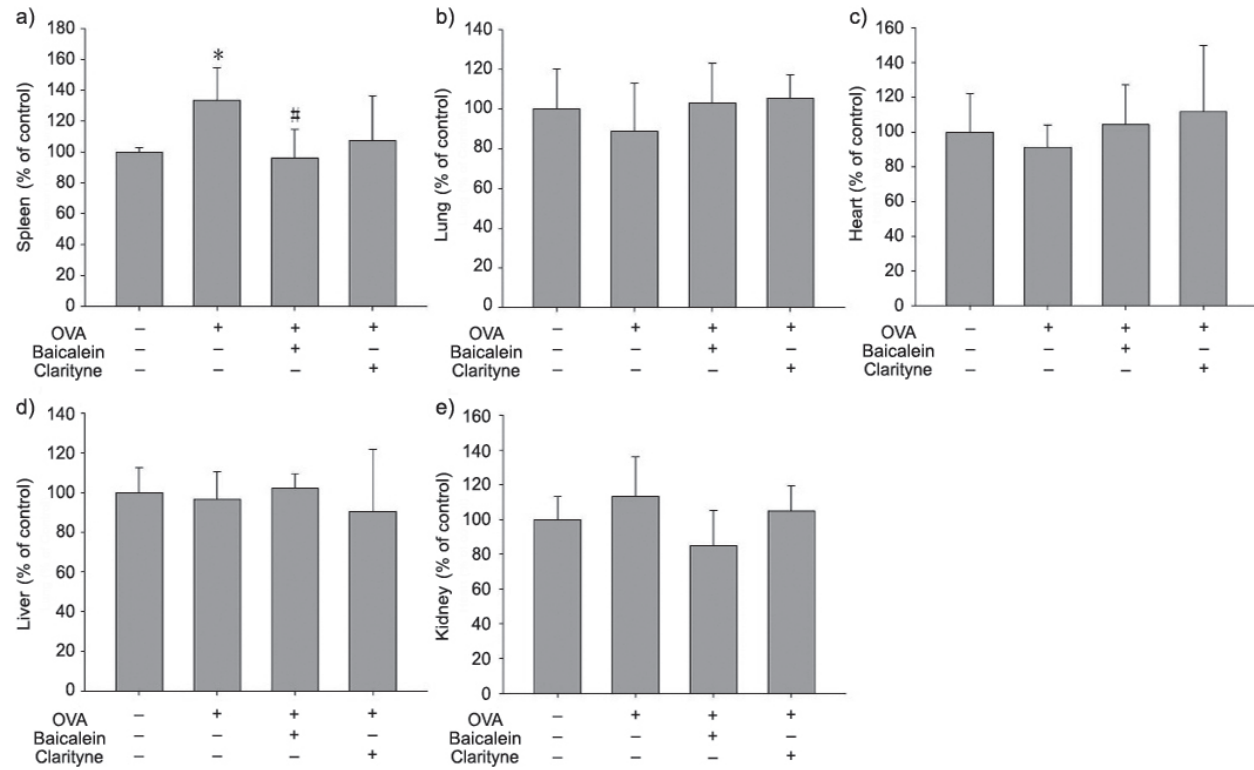

Fig. 2. Mass changes of the main organs: a) spleen, b) lung, c) heart, d) liver, e) kidney in OVA group, OVA+baicalein group, and OVA+clarityne group were measured. All data are expressed as means \pm $\mathrm{SD},{ }^{*} p<0.05$, compared to control group; $\# p<0.05$, compared to OVA group. 
a)

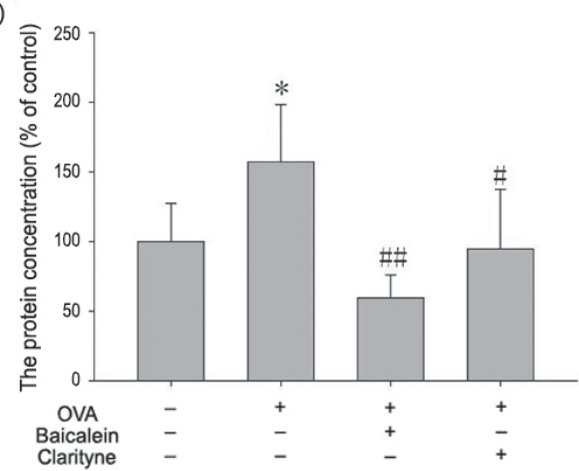

c)

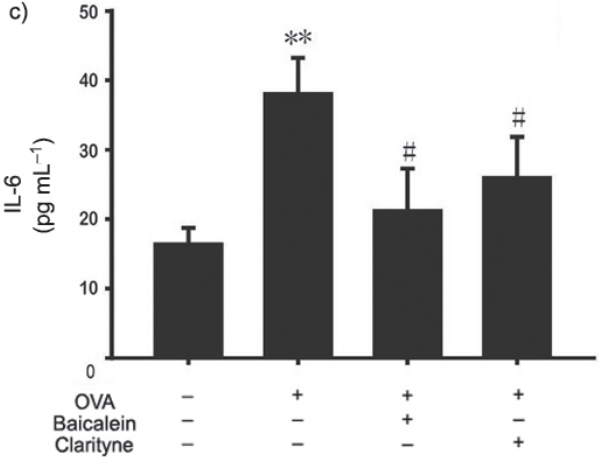

b)

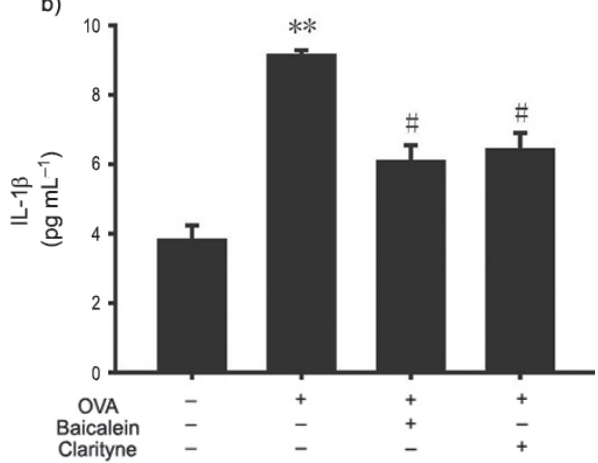

d)

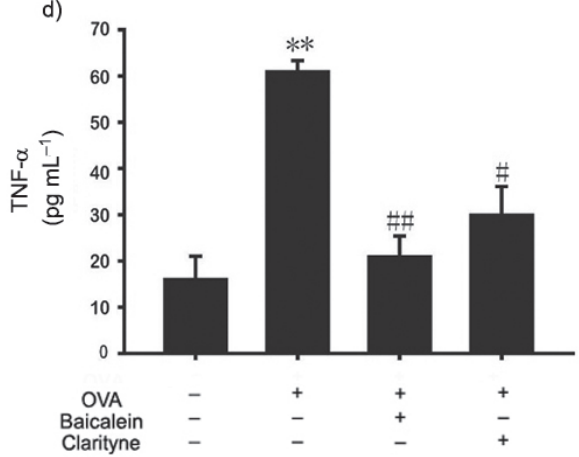

Fig. 3. Protein concentration and cytokine secretion in nasal irrigation and serum of AR rats. a) Data are expressed as means $\pm \mathrm{SD},{ }^{*} p<0.05,{ }^{* *} p<0.01$ compared to control group; $\# p<0.05$, \#\# $p<0.01$ compared to OVA group. ELISA assessment shows secretion of: b) IL-1 $\beta, \mathrm{c})$ IL-6 and d) TNF- $\alpha$ ( $n=10$ in each group). All data are expressed as means \pm SD. ${ }^{*} p<0.05,{ }^{* *} p<0.01$ compared to control group; $\# p$ $<0.05$, \#\# < 0.01 compared to OVA group.

cates that baicalein could reduce spleen damage during inflammation. In addition, no differences in other organs (heart, liver, lungs, and kidney) were found between the baicalein-treated group and the control group.

Effect of baicalein on protein concentration and the production of IL-6, TNF- $\alpha$, and $I L-1 \beta$ levels in the nasal lavage fluid and serum of $A R$ rats

Protein concentration of the AR model were significantly higher than the levels of rats in the control group, whereas those levels were significantly reduced by baicalein (Fig. 3a). To assess the adjustment effect of baicalein on the generation of inflammatory factors, the production of IL-6, TNF- $\alpha$, and IL-1 $\beta$ in the nasal lavage fluid of the AR model were measured. Relevant results are shown in Fig. $3 b-d$.

Levels of IL-6, TNF- $\alpha$, and IL-1 $\beta$ in the serum of AR-induced rats were significantly increased compared to the levels of the OVA-unsensitized rats, which were diminished by the treatment of baicalein (Fig. 4a-c). 
Effect of baicalein on inflammatory cell infiltration in both nasal mucosa tissues and lung tissues

Allergen challenge caused extensive inflammatory infiltration in the lamina propria, and the overlying epithelium was occasionally observed. Histological findings of H\&E staining in the nasal mucosa sections are shown in Fig. 5. The normal group showed no inflammatory cell infiltration in the nasal cavity, nasal septum and lateral nasal walls. In addition, the connective tissue structure was normal, both vascular congestion and proliferation disappeared, and mucosal glands and chondrocytes were normal. Notable changes were found in all histological parameters in the model group. H\&E staining indicated that the respective numbers of inflammatory cells (including eosinophils, lymphocyte and neutrophils) in the nasal mucosa of AR rats were high. The frequency of nasal inflammation was lower in the groups that received baicalein treatment and the positive group compared to the AR group. This indicated that baicalein had a marked capacity to inhibit inflammatory cell accumulation.

a)

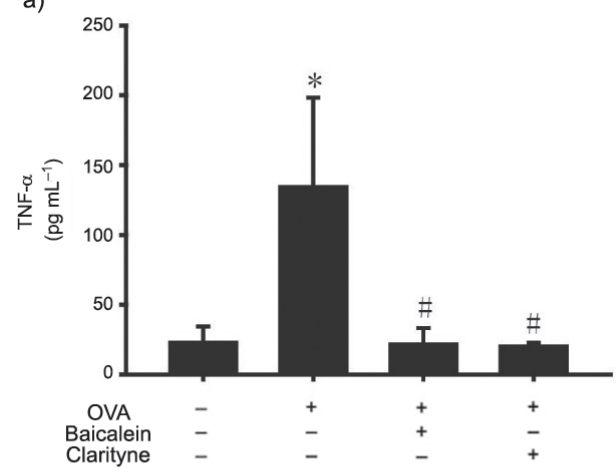

c)

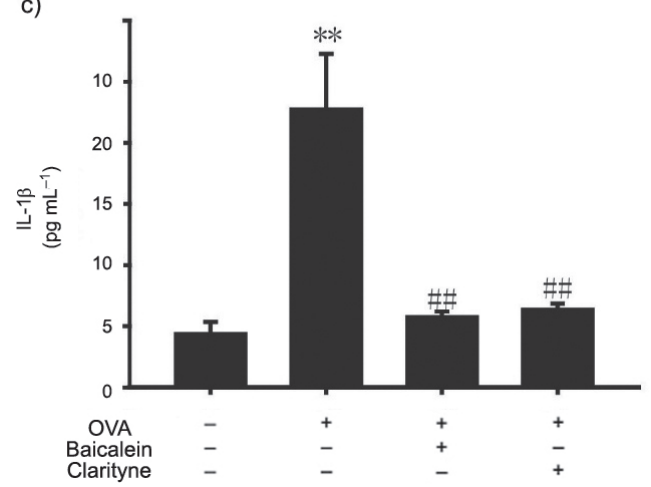

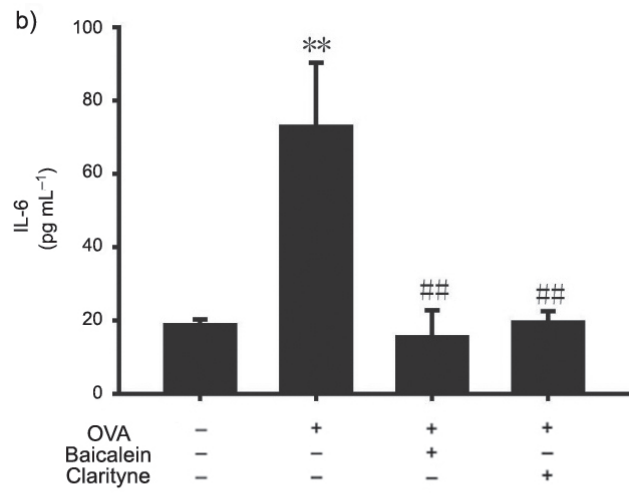

Fig. 4. ELISA assessment showed secretion of: a) TNF- $\alpha$, b) IL- 6 and c) IL-1 $\beta$ ( $n=10$ in each group). All data are expressed as means \pm SD. ${ }^{*} p<0.05,{ }^{* *} p<0.01$ compared to control group; $\# p<0.05$, \#\#p 0.01 compared to OVA group. 
Lung tissue samples were collected and the relevant sections were stained with H\&E to investigate the degree of lung injury. Lung tissues from the model group showed clear damage: interstitial edema and abscess formation, thickening of the alveolar wall and infiltration of neutrophils into the alveolar wall. However, baicalein could effectively reduce these symptoms (Fig. 6).

\section{Effect of baicalein on the expression of $p$-STAT3 in nasal mucosa tissues}

To confirm that baicalein induction was achieved by the inhibition of relevant pathways, the effect of baicalein on the STAT3 signaling pathway in the OVA-induced rats was
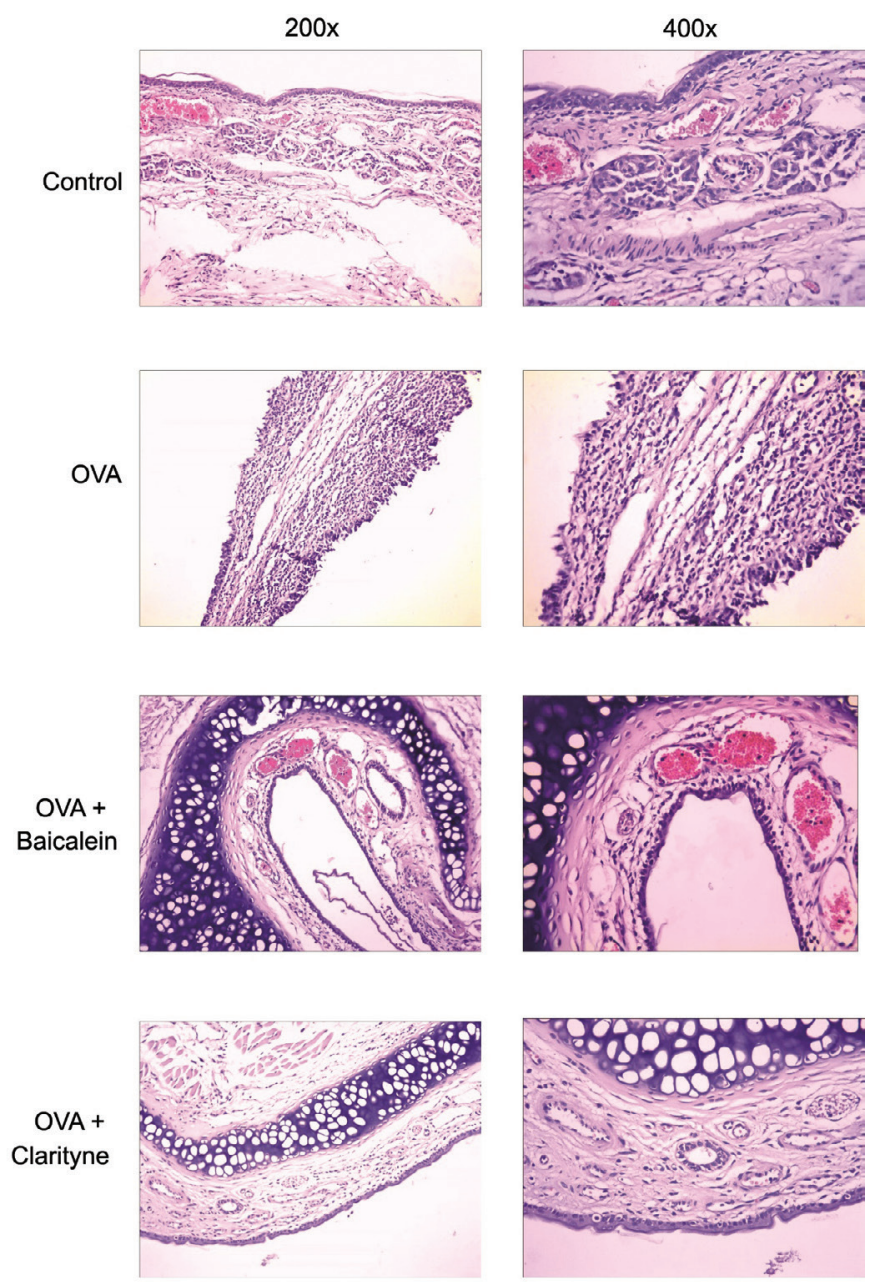

Fig. 5. Treatment with baicalein relieved lesions of nasal mucosa tissues in OVA-induced rats. Photomicrographs of H\&E stained sections of nasal mucosa from each group. 


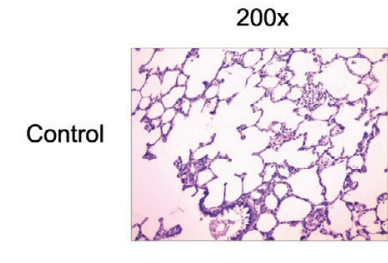

$200 x$

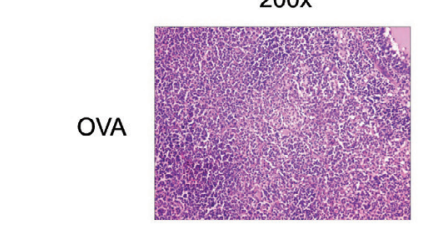

$200 x$

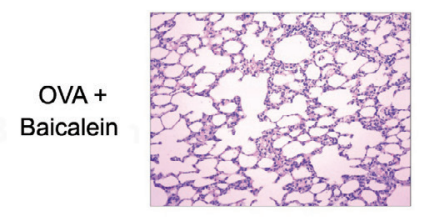

$200 x$

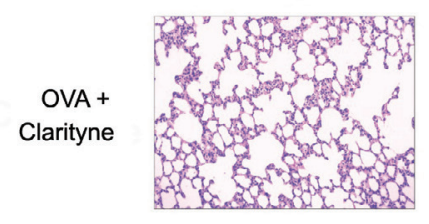

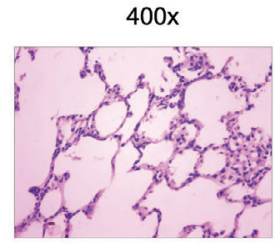

$400 x$

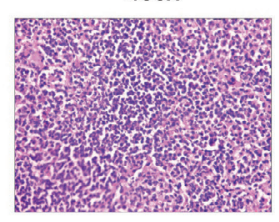

$400 x$

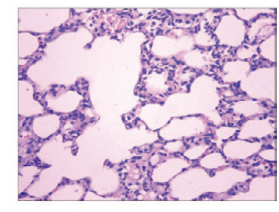

$400 x$

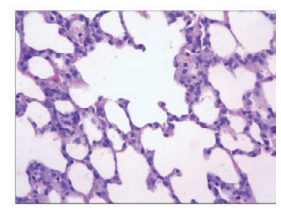

Fig. 6. Treatment with baicalein and clarityne relieved the symptoms of lung tissues in OVA-induced rats. Light photomicrographs of H\&E stained sections of lung tissue samples from each group.

investigated. The obtained results showed that baicalein inhibited the phosphorylation of STAT3 in the nasal mucosa of OVA-induced rats (Fig. 7).

$\mathrm{AR}$ is caused by an increasing number of inflammatory cytokines in lesions, which cause sneezing, rhinorrhea, and itching $(20,21)$. During the early phase of AR, the allergenIgE could induce activation of inflammatory cells, containing neutrophils, lymphocytes and eosinophils, and produce inflammation-related cytokines (including IL- 6 , TNF- $\alpha$, and IL-1 $\beta$ ). During the late-phase of AR, other inflammatory cells are added, containing eosinophils, mast cells, basophils, and T cells, in the epithelium and the deeper lamina propria (22-24). Further release of histamine, leukotrienes, chemokines and other compounds maintains the anaphylactic reaction $(6,25)$. In this study, the AR model of rats was established by repeated nasal drip OVA application, which exhibited similar allergic symptoms in humans; it can be used to analyze the detraction effect of AR (26).

In this study, typical AR symptoms were observed since day 15, which gradually disappeared after approximately $1.5 \mathrm{~h}$. The results showed that baicalein reduced the frequency of nasal itching and sneezing of rats with AR. In addition, the in vivo observation 
a)
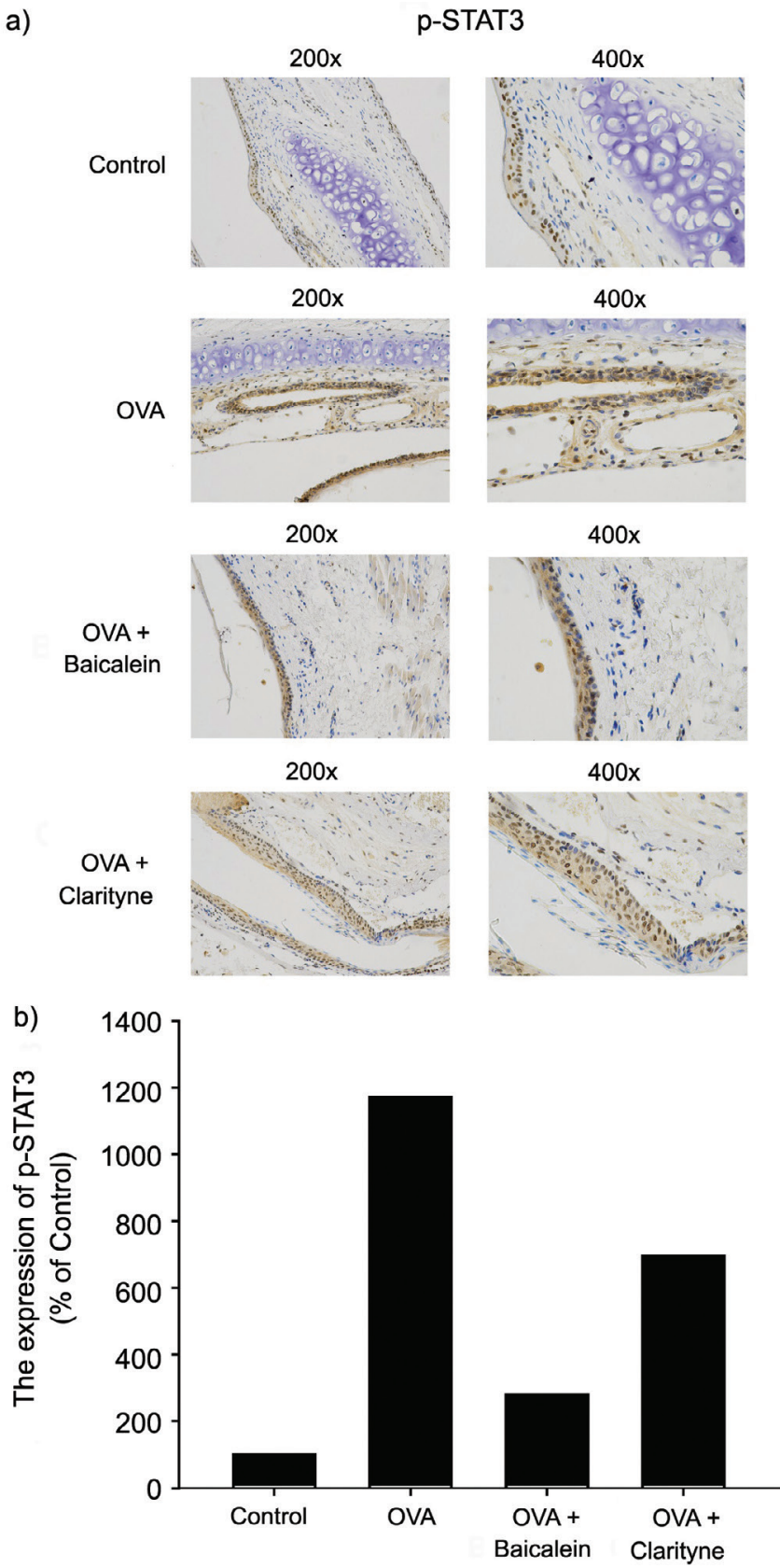

Fig. 7. Effect of baicalein on the expression of p-STAT3 in nasal mucosa tissues of OVA-induced rats. a) nuclear expression of p-STAT3 was detected using immunohistochemical staining, b) densitometric analysis of the expression of p-STAT3 protein in nasal mucosa tissues of OVA-induced rats. 
showed a strong abatement of eosinophils and infiltration of other inflammatory cells into the nasal mucosa of rats in response to baicalein treatment. This indicates that baicalein injection could effectively relieve nasal allergic symptoms. Baicalein also reversed the increase of inflammatory cytokine production in rats with AR. In summary, baicalein alleviated the allergic inflammatory reaction in this AR model.

It has been reported that STAT3 and Ah can co-regulate the differentiation of Th17 and Treg cells, and the modulation of STAT3/STAT5 activity rectifies the imbalance of Th17/Treg cells $(27,28)$. Relevant results showed that baicalein inhibited the p-STAT3 expression in the nasal mucosa of OVA-induced rats. The main limitation of this study is that the effects of baicalein on the production of Th17 and Treg cell-related cytokines (such as IL-17, IL-21, IL-22, and IL-10) were not studied to investigate whether baicalein could lead to a shift of Th17 toward Treg cells. Therefore, a future study will focus on investigating whether baicalein may regulate such a shift of Th17 and Treg cells through the STAT3 signaling pathway. Baicalein is a promising agent to alleviate the symptoms of AR and can be developed into new therapeutic agents in the future.

\section{CONCLUSIONS}

The present study demonstrated that baicalein attenuated histological alterations, aberrant tissue repair, and inflammation after OVA-induced AR. Furthermore, baicalein inhibited the generation of several inflammatory cytokines in blood and nasal lavage of rats with AR. In addition, baicalein reduced the infiltration of eosinophils, lymphocyte and neutrophils. Its action is regulated, at least in part, by a decrease of STAT3 phosphorylation and subsequent nuclear translocation.

Acknowledgments. - This work was supported by the Project Program of the Natural Science Foundation of China (No. 81603143), Shandong Medical and Health Science and Technology Development Project (No. 2017WS646, 2014WS0197), Doctoral Scientific Fund Project of Jining Medical University (No. JY2015BS08), Jining City Science and Technology Development Plan Program of Jining Science and Technology Bureau (No. 2016jn56-12) and the Teachers' Research Support Fund of Jining Medical University (No. JY2017FS009, JYFC2018KJ037).

\section{REFERENCES}

1. C. A. Tharpe and S. F. Kemp, Pediatric allergic rhinitis, Immunol. Allergy Clin. North Am. 35 (2015) 185-198; http://doi.org/10.1016/j.iac.2014.09.003

2. Y. K. Yanov, S. V. Ryazantsev, S. A. Artyushkin, I. V. Fanta and S. S. Pavlova, Allergic rhinitis: the modern aspects of therapy, Vestn. Otorinolaringol. 83 (2018) 56-60; http://doi.org/10.17116/otorino201883356

3. T. Ozdoganoglu, M. Songu and H. M. Inancli, Quality of life in allergic rhinitis, Ther. Adv. Respir. Dis. 6 (2012) 25-39; http://doi.org/10.1177/1753465811424425

4. P. W. Hellings and W. J. Fokkens, Allergic rhinitis and its impact on otorhinolaryngology, Allergy 61 (2006) 656-664; http://doi.org/10.1111/j.1398-9995.2006.01109.x

5. P. C. Bahekar, J. H. Shah, U. B. Ayer, S. N. Mandhane and R. Thennati, Validation of guinea pig model of allergic rhinitis by oral and topical drugs, Int. Immunopharmacol. 8 (2008) 1540-1551; http://doi.org/10.1016/j.intimp.2008.06.010 
6. C. Cuppari, S. Leonardi, S. Manti, M. Filippelli, T. Alterio, L. Spicuzza, L. Rigoli, T. Arrigo, V. Lougaris and C. Salpietro, Allergen immunotherapy, routes of administration and cytokine networks: an update, Immunotherapy 6 (2014) 775-786; http://doi.org/10.2217/imt.14.47

7. T. T. Bui, C. H. Piao, C. H. Song and O. H. Chai, Skullcapflavone II attenuates ovalbumin-induced allergic rhinitis through the blocking of Th2 cytokine production and mast cell histamine release, Int. Immunopharmacol. 52 (2017) 77-84; http://doi.org/10.1016/j.intimp.2017.08.029

8. P. C. Wei, L. Tong and R. Li, Effect of RORC inhibitor on HIF-1alpha and VEGF in nasal mucosa of allergic rhinitis of mice, Chin. J. Otorhinolaryngol. Head Neck Surg. 53 (2018) 751-756; http://doi. org/10.3760/cma.j.issn.1673-0860.2018.10.007

9. S. Qu, T. Qin, M. Li, S. Zhang, L. Ye, J. Wei, H. Fan and B. Chen, The effects of resiquimod in an ovalbumin-induced allergic rhinitis model, Int. Immunopharmacol. 59 (2018) 233-242; http://doi. org/10.1016/j.intimp.2018.04.015

10. J. Yao, D. Pan, Y. Zhao, L. Zhao, J. Sun, Y. Wang, Q. D. You, T. Xi, Q. L. Guo and N. Lu, Wogonin prevents lipopolysaccharide-induced acute lung injury and inflammation in mice via peroxisome proliferator-activated receptor gamma-mediated attenuation of the nuclear factor-kappaB pathway, Immunology 143 (2014) 241-257; http://doi.org/10.1111/imm.12305

11. T. T. Bui, C. H. Piao, C. H. Song, C. H. Lee, H. S. Shin and O. H. Chai, Baicalein, wogonin, and Scutellaria baicalensis ethanol extract alleviate ovalbumin-induced allergic airway inflammation and mast cell-mediated anaphylactic shock by regulation of Th1/Th2 imbalance and histamine release, Anat. Cell Biol. 50 (2017) 124-134; http://doi.org/10.5115/acb.2017.50.2.124

12. H. Yin, L. Huang, T. Ouyang and L. Chen, Baicalein improves liver inflammation in diabetic db/ $\mathrm{db}$ mice by regulating HMGB1/TLR4/NF-kappaB signaling pathway, Int. Immunopharmacol. 55 (2018) 55-62; http://doi.org/10.1016/j.intimp.2017.12.002

13. S. K. Ku and J. S. Bae, Baicalin, baicalein and wogonin inhibits high glucose-induced vascular inflammation in vitro and in vivo, BMB Rep. 48 (2015) 519-524; http://doi.org/10.5483/BMBRep.2015.48.9.017

14. T. Hong, G. B. Jin, S. Cho and J. C. Cyong, Evaluation of the anti-inflammatory effect of baicalein on dextran sulfate sodium-induced colitis in mice, Planta Med. 68 (2002) 268-271; http://doi. org/10.1055/s-2002-23143

15. X. Luo, Z. Yu, C. Deng, J. Zhang, G. Ren, A. Sun, S. Mani, Z. Wang and W. Dou, Baicalein ameliorates TNBS-induced colitis by suppressing TLR4/MyD88 signaling cascade and NLRP3 inflammasome activation in mice, Sci. Rep. 7 (2017) 16374; http://doi.org/10.1038/s41598-017-12562-6

16. C. L. Tsai, Y. C. Lin, H. M. Wang and T. C. Chou, Baicalein, an active component of Scutellaria baicalensis, protects against lipopolysaccharide-induced acute lung injury in rats, J. Ethnopharmacol. 153 (2014) 197-206; http://doi.org/10.1016/j.jep.2014.02.010

17. Z. F. Li, X. M. Xia, C. Huang, S. Zhang, J. Zhang and A. J. Zhang, Emodin and baicalein inhibit pancreatic stromal derived factor-1 expression in rats with acute pancreatitis, Hepatobiliary Pancreat. Dis. Int. 8 (2009) 201-208; http://doi.org/10.1111/j.1523-5378.2009.00663.x

18. H. G. Xin, B. B. Zhang, Z. Q. Wu, X. F. Hang, W. S. Xu, W. Ni, R. Q. Zhang and X. H. Miao, Treatment with baicalein attenuates methionine-choline deficient diet-induced non-alcoholic steatohepatitis in rats, Eur. J. Pharmacol. 738 (2014) 310-318; http://doi.org/10.1016/j.ejphar.2014.06.006

19. J. Deng, Y. Liu, H. Lee, A. Herrmann, W. Zhang, C. Zhang, S. Shen, S. J. Priceman, M. Kujawski, S. K. Pal, A. Raubitschek, D. S. Hoon, S. Forman, R. A. Figlin, J. Liu, R. Jove and H. Yu, S1PR1STAT3 signaling is crucial for myeloid cell colonization at future metastatic sites, Cancer Cell 21 (2012) 642-654; http://doi.org/10.1016/j.ccr.2012.03.039

20. X. Yuan, A. Ghosh, Q. Jie, G. He and Y. Wu, Effects of desloratadine citrate disodium injection on rat models of ovalbumin-induced allergic rhinitis: involvement of T-cell responses modulation, Int. Forum Allergy Rhinol. 5 (2015) 1170-1176; http://doi.org/10.1002/alr.21594 
21. J. Bousquet, R. Murray, D. Price, D. Somekh, L. Munter, J. Phillips and W. Czarlewski, The allergic allergist behaves like a patient, Ann. Allergy Asthma Immunol. 121 (2018) 741-742; http://doi. org/10.1016/j.anai.2018.07.034

22. X. L. Fan, Q. X. Zeng, X. Li, C. L. Li, Z. B. Xu, X. Q. Deng, J. Shi, D. Chen, S. G. Zheng and Q. L. Fu, Induced pluripotent stem cell-derived mesenchymal stem cells activate quiescent T cells and elevate regulatory $\mathrm{T}$ cell response via NF-kappaB in allergic rhinitis patients, Stem Cell Res. Ther. 9 (2018) 170; http://doi.org/10.1186/s13287-018-0896-z

23. T. Iinuma, Y. Okamoto, Y. Morimoto, T. Arai, T. Sakurai, S. Yonekura, D. Sakurai, K. Hirahara and T. Nakayama, Pathogenicity of memory Th2 cells is linked to stage of allergic rhinitis, Allergy 73 (2018) 479-489; http://doi.org/10.1111/all.13295

24. F. Xu, S. Yu, M. Qin, Y. Mao, L. Jin, N. Che, S. Liu and R. Ge, Hydrogen-rich saline ameliorates allergic rhinitis by reversing the imbalance of Th1/Th2 and up-regulation of CD4+CD25+ Foxp3+regulatory T cells, interleukin-10, and membrane-bound transforming growth factor-beta in guinea pigs, Inflammation 41 (2018) 81-92; http://doi.org/10.1007/s10753-017-0666-6

25. A. M. Watts, N. P. West, A. W. Cripps, P. K. Smith and A. J. Cox, Distinct gene expression patterns between nasal mucosal cells and blood collected from allergic rhinitis sufferers, Int. Arch. Allergy Immunol. 177 (2018) 29-34; http://doi.org/10.1159/000489609

26. M. O. Avincsal, S. Ozbal, A. O. Ikiz, C. Pekcetin and E. A. Guneri, Effects of topical intranasal doxycycline treatment in the rat allergic rhinitis model, Clin. Exp. Otorhinolaryngol. 7 (2014) 106111; http://doi.org/10.3342/ceo.2014.7.2.106

27. X. Liu, H. Hu, H. Fan, D. Zuo, Z. Shou, Y. Liao, Z. Nan and Q. Tang, The role of STAT3 and AhR in the differentiation of CD4+ T cells into Th17 and Treg cells, Medicine (Baltimore) 96 (2017) e6615; http://doi.org/10.1097/MD.0000000000006615

28. Y. Ma, X. Yuan, L. Deng, W. Xu, Y. Zheng, C. Yue, G. Zhang, F. Xie, Y. H. Yang, M. P. Gantier, J. Liu, D. Xu and L. Shen, Imbalanced frequencies of Th17 and Treg cells in acute coronary syndromes are mediated by IL-6-STAT3 signaling, PLoS One 8 (2013) e72804; http://doi.org/10.1371/journal. pone.0072804 